\title{
Hypertension, Periodontal Disease, and Potassium Intake in Nonsmoking, Nondrinker African Women on No Medication
}

\author{
Masashi Yamori, ${ }^{1}$ Marina Njelekela, ${ }^{2}$ Jacob Mtabaji, ${ }^{3}$ Yukio Yamori, ${ }^{4}$ and Kazuhisa Bessho ${ }^{1}$ \\ ${ }^{1}$ Department of Oral and Maxillofacial Surgery, Graduate School of Medicine, Kyoto University, 54 Shogoin-Kawahara-cho, Sakyo-ku, \\ Kyoto 606-8507, Japan \\ ${ }^{2}$ Department of Physiology, School of Medicine, Muhimbili University of Health and Allied Sciences (MUHAS), P.O. Box 65001, \\ Dar es Salaam, Tanzania \\ ${ }^{3}$ Department of Physiology, Weill Bugando University College of Health Sciences, P.O. Box 1464, Mwanza, Tanzania \\ ${ }^{4}$ Mukogawa Women's University Institute for World Health Development, 4-16 Edagawa-cho, Nishinomiya, Hyogo 663-8143, Japan
}

Correspondence should be addressed to Masashi Yamori, yamori@kuhp.kyoto-u.ac.jp

Received 14 February 2011; Revised 5 May 2011; Accepted 15 June 2011

Academic Editor: Zafar Israili

Copyright (C) 2011 Masashi Yamori et al. This is an open access article distributed under the Creative Commons Attribution License, which permits unrestricted use, distribution, and reproduction in any medium, provided the original work is properly cited.

The purpose of this cross-sectional study was to investigate the association of periodontitis and/or tooth loss with hypertension by excluding the common confounders. Eighty-one Tanzanian women who were aged 46-58 years, nonsmokers, nonalcoholic drinkers, and on no medication underwent clinical examination. Multiple-regression analysis showed that the severity of periodontitis was significantly correlated with increased systolic blood pressure and diastolic blood pressure. Simple-regression analysis indicated that the severity of periodontitis was inversely correlated with 24-hour urinary excretion of potassium $(r=$ $-0.579, P=0.0004)$ and also inversely with the frequency of intakes of green vegetables $(r=-0.232, P=0.031)$ and fruits $(r=-0.217, P=0.0043)$. Low-potassium intake in the diet mostly accompanied by low dietary fiber intake increases BP as well as periodontal inflammation. Potassium intake may be an important factor linking periodontitis and hypertension in middleaged nonsmoking and nonalcoholic women on no medication, although chronic inflammation such as periodontitis may cause hypertension through a more direct mechanism.

\section{Introduction}

Periodontitis, which affects a large number of adults globally, is epidemiologically related to atherosclerotic vascular diseases and metabolic syndrome $[1,2]$. Periodontitis as chronic inflammation destroys the supporting structure of the teeth and increases the level of C-reactive protein (CRP) [35]. Recent attention has focused on elevated serum CRP, a marker of systemic inflammation, as a strong and independent risk factor or predictor of hypertension (HT) [6]. The systemic response to periodontal infection is a possible pathway underlying the observed association between periodontitis and increased risk for HT.

Epidemiological systematic examinations about the noted relationship between periodontitis and vascular diseases should be conducted among healthy subjects who have never smoked [7] because smoking is regarded as a strong confounder and may spuriously inflate the association between periodontitis and vascular diseases. Alcohol consumption is also one of the risk factors that can lead to the development of not only HT [8] but also periodontitis [9]. In nonsmoking menopausal Japanese women, tooth loss but not periodontitis was proven to be significantly associated with an increased risk of HT [10]. Medical care is also a confounding variable that strongly affects epidemiological studies in developed countries such as Japan, where epidemiological analysis on the association between vascular diseases and oral health may be complicated by the common use of certain antihypertensive medications that affect not only blood pressure (BP) but also oral health status [11]. In order to avoid these confounders, we selected middle-aged African women on no medication, who were nonsmokers and did not consume 
TABLE 1: Basic characteristics of participants.

\begin{tabular}{lc}
\hline Parameter & Women $(n=81)$ \\
\hline Age (year) & $52.09 \pm 3.92$ \\
SBP (mmHg) & $139.93 \pm 26.60$ \\
DBP (mmHg) & $80.33 \pm 20.80$ \\
BMI & $28.57 \pm 6.69$ \\
Total-C & $5.33 \pm 1.23$ \\
TG & $1.63 \pm 0.93$ \\
HDL- C & $1.34 \pm 0.39$ \\
HbAlc & $5.22 \pm 1.43$ \\
Vegetables (days/week) & $4.20 \pm 2.32$ \\
Fruit (days/week) & $3.74 \pm 1.47$ \\
Periodontitis & $2.37 \pm 0.66$ \\
Tooth loss & $3.70 \pm 3.81$ \\
\hline
\end{tabular}

Values are presented as mean \pm standard deviation (SD). BMI: Body Mass Index (body weight $/ \mathrm{height}^{2}, \mathrm{~kg} / \mathrm{m}^{2}$ ), Total-C: serum total cholesterol $(\mathrm{mmol} / \mathrm{L})$, TG: triglycerides (mmol/L), HDL-C: high-density-lipoprotein cholesterol (mmol/L), and HbAlc: glycosylated hemoglobin (\%). Vegetable and fruit intakes are expressed as the number of days per week when they were consumed. Periodontitis: the average CPTIN of each tooth. Tooth loss: number of teeth lost.

alcoholic drinks, and investigated the possible association of periodontitis and tooth loss with the risk factors for HT in the present study.

\section{Methods}

2.1. Study Design and Subjects. The present study was conducted in Tanzania according to the protocol for Cardiovascular Diseases and Alimentary Comparison (CARDIAC) Study coordinated by the World Health Organization (WHO) [12-14]. We previously reported associations between $\mathrm{HT}$ and its traditional risk factors such as body mass index (BMI), salt intake, and $\mathrm{Na} / \mathrm{K}$ ratio in the CARDIAC study in Tanzania [14-18]. One hundred women aged 4658, living in Temeke, Dar es Salaam, were randomly selected from an administrative list and invited to participate in the study by letter. Detailed oral examination was limited to women because of the higher prevalence of smokers and alcohol drinkers among men, who were therefore considered not suitable for analysis of the association of oral health with dietary factors [7]. Eighty-one women responded to the invitation for BP and anthropometrical measurements, 24hour urine collection, blood sampling, dental examination, and lifestyle questionnaires. This study was approved by the Institutional Ethical Review Board of Muhimbili University College of Health Science. Written informed consent was obtained in accordance with the institutional rules.

2.2. Health Examination. In order to eliminate observer bias, $\mathrm{BP}$ was measured using a centrally calibrated automatic BP measurement system used for the CARDIAC Study (Khi machine) $[12,13]$. BP was measured 3 times for each subject, and the average of the 3 measurements was used in this analysis. Blood was sampled after a $10-14 \mathrm{~h}$ fast. Urinary bags (U-container N, Ono Medical Company Osaka, Japan) were used for collecting 24-hour urine and the validity was confirmed by checking creatinine excretion [13]. Blood and urine samples for the CARDIAC Study were analyzed centrally at the former WHO Collaborating Center for Research on Primary Prevention of Cardiovascular Diseases, Graduate School of Human and Environmental Studies, Kyoto University, Kyoto, Japan. Details of other health examination methods were described in previous reports on the protocol and findings of the CARDIAC study in Tanzania [14].

2.3. Oral Examination. A single examiner (M.Y.) carried out, without knowing other health-related data, the oral examination including tooth count and the assessment of periodontitis, which was measured according to the WHO community periodontal index of treatment needs (CPITN). The specially designed WHO periodontal probe with a sensing force of not over $20 \mathrm{~g}$ was utilized. The ten teeth examined were $17,16,11,26,27,47,46,31,36$, and 37 ; for each tooth, the highest index found was recorded according to the following scale: (0) periodontal health; (1) gingival bleeding; (2) calculus detected during probing; (3) pocket 4 to $5 \mathrm{~mm}$ deep; and (4) pocket $6 \mathrm{~mm}$ deep and over. Periodontal condition was reported as the average CPTIN condition of each tooth, the scoring for which is well established and regarded as the global standard measurement for epidemiological and screening studies on periodontitis [19-21].

2.4. Statistical Analysis. Data were analyzed using the program Statview 5 for microcomputers from the SAS Institute Inc. Simple correlation analysis using the Pearson method allowed the assessment of univariate relationships. The variables were regarded as normally distributed.

\section{Results}

In this study, we assessed the association of tooth loss and periodontitis with $\mathrm{BP}$, and the traditional risk factors of HT in 81 women aged 46-58 years, whose basic characteristics are shown in Table 1. None of the subjects in this population was found to be using or have used drugs that affect lipid metabolism, BP, or blood sugar. Additionally, they had never smoked and never drunk alcohol because of their local Islamic religious discipline and culture.

In multiple-regression analysis, the severity of periodontitis was significantly correlated with systolic BP (SBP) $(r=$ $0.288, P=0.018)$ and diastolic BP (DBP) $(r=0.293, P=$ 0.015 ), and tooth loss (the number of teeth missing) was also significantly correlated with SBP $(r=0.308, P=0.010)$ and $\operatorname{DBP}(r=0.417, P=0.0005)$ (Table 2$)$. These results indicate that periodontitis and tooth loss are significantly and independently associated with increases in BP in nonsmoking middle-aged Tanzanian women. In addition, to assess the association between HT and periodontitis, participants were divided into 3 groups. The severely hypertensive (SBP $>180$ or DBP > 110) group had a significant difference in the severity of periodontitis (CPITN, $2.82 \pm 0.64$; mean \pm standard deviation (SD)) from the normal to borderline hypertensive BP $($ SBP $<160$ or DBP $<100)$ group $(2.29 \pm 0.61, P<0.05)$. 
TABLE 2: Multiple-regression analysis of BP, periodontitis, tooth loss, and traditional risk factors possibly related to hypertension in middleaged women.

\begin{tabular}{lcccc}
\hline \multirow{2}{*}{ Parameter } & \multicolumn{2}{c}{ Systolic blood pressure $(\mathrm{mmHg})$} & \multicolumn{2}{c}{ Diastolic blood pressure $(\mathrm{mmHg})$} \\
& $\beta$ & $P$ value & $\beta$ & $P$ value \\
\hline Age (year) & 0.103 & 0.364 & 0.051 & 0.649 \\
BMI & 0.270 & $0.024^{\dagger}$ & 0.299 & $0.012^{\dagger}$ \\
Total-C & 0.074 & 0.602 & 0.125 & 0.374 \\
TG & 0.187 & 0.545 & 0.099 & 0.452 \\
HDL-C & 0.081 & 0.165 & -0.056 & 0.668 \\
HbAlc & 0.259 & 0.190 & 0.139 & 0.194 \\
Periodontitis & 0.288 & $0.018^{\dagger}$ & 0.293 & $0.015^{\dagger}$ \\
Tooth loss & 0.308 & $0.010^{\dagger}$ & 0.417 & $0.0005^{\ddagger}$ \\
\hline
\end{tabular}

Values are regression coefficients $(\beta)$ and $P$ values. ${ }^{\dagger} P<0.05,{ }^{\ddagger} P<0.001$.

Multiple correlation coefficient $|R|=0.609$ for systolic blood pressure, $|R|=0.621$ for diastolic blood pressure.

TABLE 3: Simple correlation coefficients between periodontitis and tooth loss with traditional risk factors possibly related to hypertension in middle-aged women.

\begin{tabular}{|c|c|c|c|c|c|}
\hline \multicolumn{3}{|c|}{ Periodontitis } & \multicolumn{3}{|c|}{ Tooth loss } \\
\hline Versus & $r$ & $P$ value & Versus & $r$ & $P$ value \\
\hline $\mathrm{NaCl}(\mathrm{g} / \text { day })^{\dagger}$ & -0.179 & 0.329 & $\mathrm{NaCl}$ (g/day) & -0.112 & 0.547 \\
\hline $\mathrm{KCl}(\mathrm{g} / \text { day })^{\dagger}$ & -0.579 & $0.0004^{\S}$ & $\mathrm{KCl}$ (g/day) & -0.204 & 0.265 \\
\hline $\mathrm{Na} / \mathrm{K}$ & 0.160 & 0.385 & $\mathrm{Na} / \mathrm{K}$ & 0.081 & 0.660 \\
\hline $\mathrm{Mg}$ (mg/day) & -0.336 & 0.060 & $\mathrm{Mg}$ (mg/day) & -0.273 & 0.131 \\
\hline Total-C & 0.002 & 0.986 & Total-C & -0.102 & 0.400 \\
\hline TG & -0.007 & 0.951 & TG & -0.083 & 0.495 \\
\hline HDL-C & 0.059 & 0.627 & HDL-C & -0.114 & 0.345 \\
\hline BMI & -0.160 & 0.184 & BMI & -0.271 & $0.022^{\ddagger}$ \\
\hline $\mathrm{HbAlc}$ & -0.071 & 0.558 & HbAlc & -0.158 & 0.189 \\
\hline
\end{tabular}

Values are correlation coefficients $(r),{ }^{\ddagger} P<0.05,{ }^{\S} P<0.001$.

24-hour urine $(n=32)$, blood chemistry and BMI $(n=81)$, and ${ }^{\dagger} 24$-hour urinary sodium and potassium excretions were measured and the intakes were estimated as the amount of chloride salt.

Next, we tested the association of periodontitis and tooth loss with traditional risk factors for HT in women (Table 3). There was a strong inverse correlation of 24-hour urinary excretion of potassium ( $Y$; amount estimated as $\mathrm{KCL})$ with severity of periodontitis $(X)(Y=3.144-.433 X$; $r=-0.579$, and $\left.r^{2}=0.34, P<0.0004\right)$, but not with tooth loss. No other risk factors were correlated significantly with periodontitis. On the other hand, tooth loss was significantly correlated with BMI $(r=-0.271, P=0.022)$ but not with the 24-hour urinary excretion of potassium. Since 24-hour urinary excretion was closely related to dietary intake of potassium-rich vegetables and fruit [22], these results suggest that nutritional factors, particularly potassium intake, were associated with periodontitis but not with tooth loss.

Therefore, we further assessed the relationship between dietary factors and periodontitis. There were significant negative correlations of the intakes of green vegetables $(r=-0.232, P=0.031)$ and fruit $(r=-0.217, P=0.043)$ with the severity of periodontitis in women (Table 4). These results indicate that the intake of potassium-rich foods, such as green vegetables and fruit, is associated with periodontitis. The intake of skimmed milk, but not whole milk, was significantly inversely correlated with the severity of periodontitis. This might be related to the preference for skimmed milk among vegetarians.

\section{Discussion}

In this study, we demonstrated that periodontitis and tooth loss were significantly associated with an increased risk of HT in middle-aged African women on no medication without any direct influence of smoking and drinking alcohol. HT is associated with increased levels of the markers of inflammation, including CRP and proinflammatory cytokines [6]. Periodontitis as chronic bacterial infection by Gramnegative bacteria is also associated with increased levels of inflammation-related markers such as CRP in circulation [3-5]. Recent studies suggest that everyday events such as chewing and brushing of teeth contribute more significantly to the cumulative exposure of the vascular system to oral bacteria [23]. Periodontal pathogens were identified in carotid atheromatous plaques obtained by endarterectomy from patients [24]. In periodontitis, bacteremia and/or endotoxemia may trigger a systemic inflammatory response that, in turn, may cause endothelial dysfunction and thus increase BP and accelerate atherosclerosis. 
TABLE 4: Simple correlation coefficients between periodontitis and dietary intakes expressed by the frequency or the amount.

\begin{tabular}{lcc}
\hline & \multicolumn{2}{c}{ Periodontitis } \\
Versus & $r$ & $P$ value \\
\hline Vegetables (days/week) & -0.232 & $0.031^{\dagger}$ \\
Fruit (days/week) & -0.217 & $0.043^{\dagger}$ \\
Skimmed milk (mL/day) & -0.304 & $0.004^{\dagger}$ \\
Whole milk (mL/day) & -0.097 & 0.375 \\
Coconut milk (mL/day) & 0.119 & 0.273 \\
Meat (days/week) & 0.058 & 0.598 \\
Fish (days/week) & 0.052 & 0.637 \\
\hline
\end{tabular}

Values are correlation coefficients $(r),{ }^{\dagger} P<0.05$.

One possible pathway is that periodontal infection induces systemic inflammation contributing to the development of HT. Endothelial dysfunction through periodontal infection-inflammation pathway $[3,25]$ might be the link between periodontitis and HT. The emerging evidence on the positive association of other inflammatory disorders, such as systemic lupus erythematosus [26] and rheumatoid arthritis [27], with increased HT risk, supports this as a plausible mechanism. The second pathway is that periodontitis might be associated with HT-related nutrients and foods. In this study, the severity of periodontitis but not the number of teeth lost was significantly and strongly associated only with daily potassium intake among the traditional risk factors related to HT, including nutrients, cholesterols, obesity, and diabetes. Moreover, we found that periodontitis was associated with less intake of potassium-containing foods, fruit, and vegetables. These results suggest that high consumption of potassium, possibly reducing the risk of HT, might decrease periodontitis severity and partially explain the association between periodontitis and HT.

The mechanisms on the possible causative links of periodontitis with potassium intake could be speculated. Firstly, potassium intake checked by 24-hour urinary excretion is associated with the consumption of vegetables and fruit [22], which may beneficially influence oral health [28]. Secondly, the tissue destruction in periodontitis is considered to be caused mainly by an aberrant inflammatory response involving prolonged release of neutrophil enzymes and reactive oxygen species. Potassium is reported to inhibit reactive oxygen species formation by human white blood cells [29] as well as to protect against hypertensive vascular injury and cardiac dysfunction, though, reducing reactive oxygen species in salt-induced experimental hypertension [30]. Vegetables and fruit are primary dietary sources of antioxidants, such as vitamins $C$ and $E$. Intake of potassium-rich vegetables and fruit might help to prevent periodontitis by reducing free radicals and oxidative stress in periodontal tissue.

Previously, we reported that the mean levels of 8-hydroxydeoxyguanosine (8-OHdG), a marker of oxidative DNA damage in 24-hour urine samples, were significantly higher in hypertensive subjects than in normotensive subjects in Tanzania [31]. Our results suggested that a beneficial effect of the intake of potassium-rich vegetables and fruit on HT and periodontitis might be related to their roles in inflammation or oxidative stress, and that 24-hour urinary excretion of potassium might be a good biomarker for the risk evaluation of periodontitis as well as HT. The possibility that people with severe periodontitis who lose more teeth tend to eat less vegetables to absorb potassium, and therefore have higher BP, may be excluded because 24-hour urinary potassium excretion was significantly inversely related only with periodontitis but not with tooth loss.

Several limitations in the present study should be considered. First, the cross-sectional design basically precludes any causal inferences about the role of potassium intake between periodontitis and HT. Second, our analysis was carried out only once. Nevertheless, this study has several strengths. First, we controlled other major traditional risk factors, including potential confounders such as age, gender, race, diabetes, hypercholesterolemia, BMI, smoking, and alcohol consumption. The age range was limited to 4658 years old to avoid the aging influence on HT and periodontitis. Second, the subjects were particularly suitable for the investigation of dietary influence on BP and oral health because they were on no medication and neither consumed alcohol nor smoked. Both of these are well-known confounders causing periodontitis as well as risk factors of HT. Third, to avoid examiner bias, oral examination was performed by a single examiner who was unaware of other health examination data. In addition, to provide an adequate assessment of the severity of periodontitis, CPITN was used as recommended by the WHO. CPITN score has become widely accepted as the method of choice for epidemiological and screening studies for periodontitis [19-21]. Fourth, nutritional assessments were carried out using not only a food frequency questionnaire but also biomarkers from 24hour urine samples from African Muslim women whose dietary customs did not vary on a daily basis.

\section{Conclusion}

The present study demonstrated the association among dietary potassium intake, periodontitis, and HT. Low-potassium intake in the diet mostly accompanied by low dietary fiber intake increases BP as well as periodontal inflammation. Moreover, periodontitis may raise BP through chronic inflammatory and oxidative stress mechanisms. Therefore, oral health is important in reducing the risk of HT, and traditional dietary customs of consuming more potassium from vegetables and fruit are expected to decrease the risk of HT and periodontitis, both presently deteriorating the quality of life in the elderly, although this expectation remains to be proven by long-term intervention trials.

\section{Acknowledgments}

The authors are grateful to all those who participated in the CARDIAC health survey and oral health examination and to volunteer workers from the study sites and from the former WHO collaborating Center for Research on Primary Prevention of Cardiovascular Disease. This study was supported in part by the CARDIAC Study fund donated to the WHO 
through the Japan Heart Foundation from over 300,000 Japanese people and by Grant-in-Aid for International Research from the Japanese Government.

\section{References}

[1] F. DeStefano, R. F. Anda, H. S. Kahn, D. F. Williamson, and C. M. Russell, "Dental disease and risk of coronary heart disease and mortality," British Medical Journal, vol. 306, no. 6879, pp. 688-691, 1993.

[2] Y. Shimazaki, T. Saito, K. Yonemoto, Y. Kiyohara, M. Iida, and Y. Yamashita, "Relationship of metabolic syndrome to periodontal disease in Japanese women: the Hisayama study," Journal of Dental Research, vol. 86, no. 3, pp. 271-275, 2007.

[3] G. Seinost, G. Wimmer, M. Skerget et al., "Periodontal treatment improves endothelial dysfunction in patients with severe periodontitis," American Heart Journal, vol. 149, no. 6, pp. 1050-1054, 2005.

[4] K. J. Joshipura, H. C. Wand, A. T. Merchant, and E. B. Rimm, "Periodontal disease and biomarkers related to cardiovascular disease," Journal of Dental Research, vol. 83, no. 2, pp. 151-155, 2004.

[5] F. D'Aiuto, M. Parkar, G. Andreou et al., "Periodontitis and systemic inflammation: control of the local infection is associated with a reduction in serum inflammatory markers," Journal of Dental Research, vol. 83, no. 2, pp. 156-160, 2004.

[6] H. D. Sesso, J. E. Buring, N. Rifai, G. J. Blake, J. M. Gaziano, and P. M. Ridker, "C-reactive protein and the risk of developing hypertension," Journal of the American Medical Association, vol. 290, no. 22, pp. 2945-2951, 2003.

[7] P. P. Hujoel, "Does chronic periodontitis cause coronary heart disease? A review of the literature," The Journal of the American Dental Association, vol. 133, supplement 1, pp. 31S-36S, 2002.

[8] A. Ascherio, C. Hennekens, W. C. Willett et al., "Prospective study of nutritional factors, blood pressure, and hypertension among US women," Hypertension, vol. 27, no. 5, pp. 10651072, 1996.

[9] W. Pitiphat, A. T. Merchant, E. B. Rimm, and K. J. Joshipura, "Alcohol consumption increases periodontitis risk," Journal of Dental Research, vol. 82, no. 7, pp. 509-513, 2003.

[10] A. Taguchi, M. Sanada, Y. Suei et al., "Tooth loss is associated with an increased risk of hypertension in postmenopausal women," Hypertension, vol. 43, no. 6, pp. 1297-1300, 2004.

[11] M. Harel-Raviv, M. Eckler, K. Lalani, E. Raviv, and M. Gornitsky, "Nifedipine-induced gingival hyperplasia. A comprehensive review and analysis," Oral Surgery, Oral Medicine, Oral Pathology, Oral Radiology and Endodontology, vol. 79, no. 6, pp. 715-722, 1995.

[12] "Excerpts from the WHO CARDIAC Study Protocol," Journal of Cardiovascular Pharmacology, vol. 16, supplement 8, pp. 7577, 1990.

[13] Y. Yamori, L. Liu, K. Ikeda et al., "Distribution of twentyfour hour urinary taurine excretion and association with ischemic heart disease mortality in 24 populations of 16 countries: results from the WHO-CARDIAC Study," Hypertension Research, vol. 24, no. 4, pp. 453-457, 2001.

[14] M. Njelekela, H. Negishi, Y. Nara et al., "Cardiovascular risk factors in Tanzania: a revisit," Acta Tropica, vol. 79, no. 3, pp. 231-239, 2001.

[15] M. Njelekela, K. Ikeda, J. Mtabaji, and Y. Yamori, "Dietary habits, plasma polyunsaturated fatty acids and selected coronary disease risk factors in Tanzania," East African Medical Journal, vol. 82, no. 11, pp. 572-578, 2005.
[16] J. P. Mtabaji, Y. Nara, and Y. Yamori, "The cardiac study in Tanzania: salt intake in the causation and treatment of hypertension," Journal of Human Hypertension, vol. 4, no. 2, pp. 8081, 1990.

[17] J. P. Mtabaji, Y. Nara, Y. Moriguchi, and Y. Yamori, "Diet and hypertension in Tanzania," Journal of Cardiovascular Pharmacology, vol. 16, supplement 8, pp. S3-S5, 1990.

[18] M. Njelekela, T. Sato, Y. Nara et al., "Nutritional variation and cardiovascular risk factors in Tanzania-rural-urban difference," South African Medical Journal, vol. 93, no. 4, pp. 295299, 2003.

[19] F. Angeli, P. Verdecchia, C. Pellegrino et al., "Association between periodontal disease and left ventricle mass in essential hypertension," Hypertension, vol. 41, no. 3, pp. 488-492, 2003.

[20] S. J. Janket, M. Qvarnström, J. H. Meurman, A. E. Baird, P. Nuutinen, and J. A. Jones, "Asymptotic dental score and prevalent coronary heart disease," Circulation, vol. 109, no. 9, pp. 1095-1100, 2004.

[21] T. Schillinger, W. Kluger, M. Exner et al., "Dental and periodontal status and risk for progression of carotid atherosclerosis: the inflammation and carotid artery risk for atherosclerosis study dental substudy," Stroke, vol. 37, no. 9, pp. 2271-2276, 2006.

[22] J. Tamaki, K. Yoshita, Y. Kikuchi et al., "Applicability of the stages of change model for analyzing fruit and vegetable intake in relation to urinary potassium excretion: baseline results from the High-Risk and Population Strategy for Occupational Health Promotion (HIPOP-OHP) study," Hypertension Research, vol. 27, no. 11, pp. 843-850, 2004.

[23] L. Forner, T. Larsen, M. Kilian, and P. Holmstrup, "Incidence of bacteremia after chewing, tooth brushing and scaling in individuals with periodontal inflammation," Journal of Clinical Periodontology, vol. 33, no. 6, pp. 401-407, 2006.

[24] V. I. Haraszthy, J. J. Zambon, M. Trevisan, M. Zeid, and R. J. Genco, "Identification of periodontal pathogens in atheromatous plaques," Journal of Periodontology, vol. 71, no. 10, pp. 1554-1560, 2000.

[25] M. S. Tonetti, F. D’Aiuto, L. Nibali et al., "Treatment of periodontitis and endothelial function," New England Journal of Medicine, vol. 356, no. 9, pp. 911-920, 2007.

[26] F. Selzer, K. Sutton-Tyrrell, S. Fitzgerald, R. Tracy, L. Kuller, and S. Manzi, "Vascular stiffness in women with systemic lupus erythematosus," Hypertension, vol. 37, no. 4, pp. 10751082, 2001.

[27] M. J. Roman, R. B. Devereux, J. E. Schwartz et al., "Arterial stiffness in chronic inflammatory diseases," Hypertension, vol. 46, no. 1, pp. 194-199, 2005.

[28] M. S. Al-Zahrani, "Increased intake of dairy products is related to lower periodontitis prevalence," Journal of Periodontology, vol. 77, no. 2, pp. 289-294, 2006.

[29] R. D. McCabe, M. A. Bakarich, K. Srivastava, and D. B. Young, "Potassium inhibits free radical formation," Hypertension, vol. 24, no. 1, pp. 77-82, 1994.

[30] M. Kido, K. Ando, M. L. Onozato et al., "Protective effect of dietary potassium against vascular injury in salt-sensitive hypertension," Hypertension, vol. 51, no. 2, pp. 225-231, 2008.

[31] H. Negishi, K. Ikeda, S. Kuga et al., "The relation of oxidative DNA damage to hypertension and other cardiovascular risk factors in Tanzania," Journal of Hypertension, vol. 19, no. 3, pp. 529-533, 2001. 


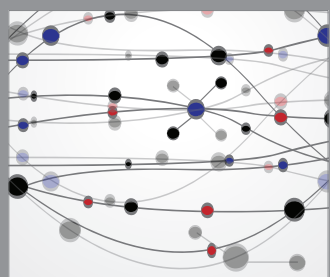

The Scientific World Journal
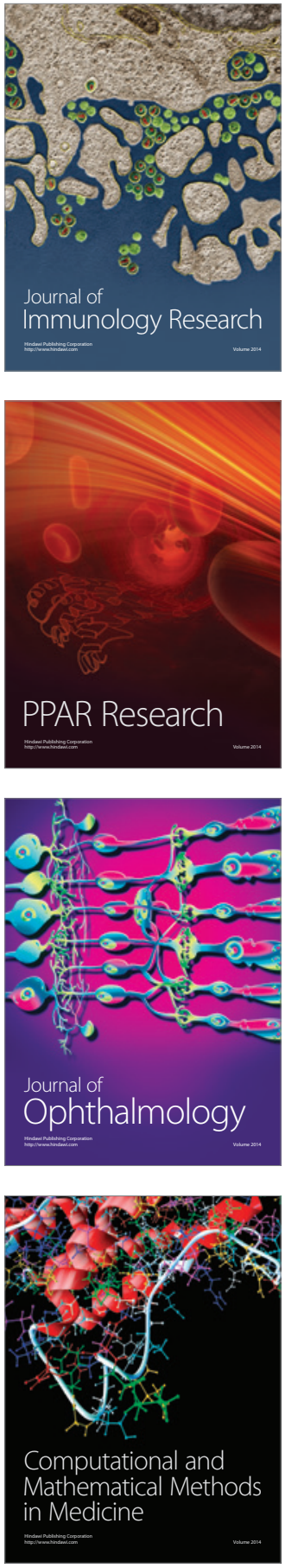

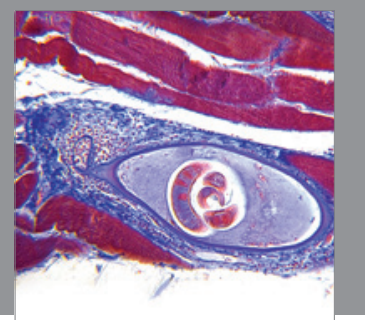

Gastroenterology

Research and Practice
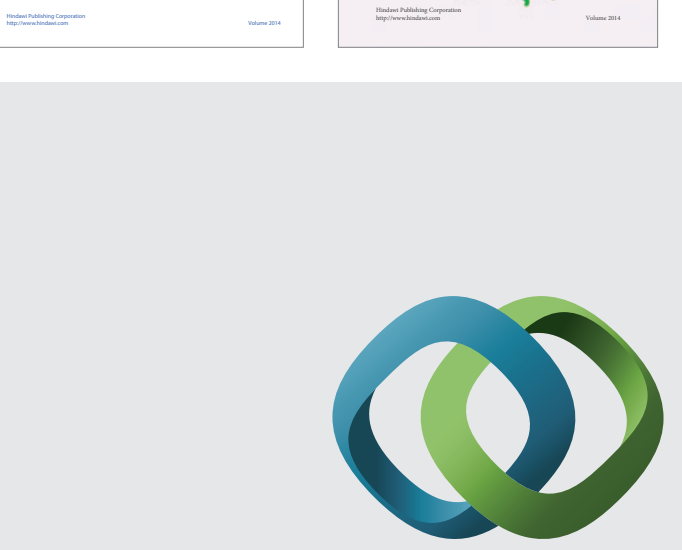

\section{Hindawi}

Submit your manuscripts at

http://www.hindawi.com
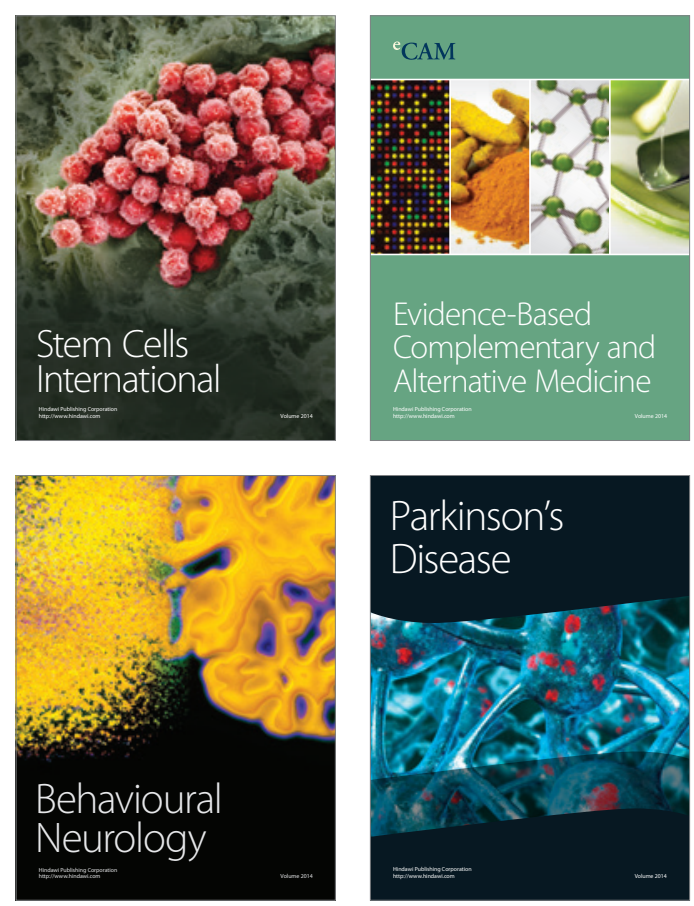

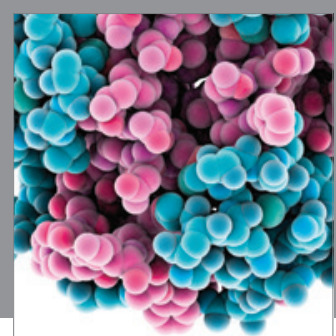

Journal of
Diabetes Research

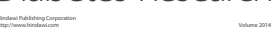

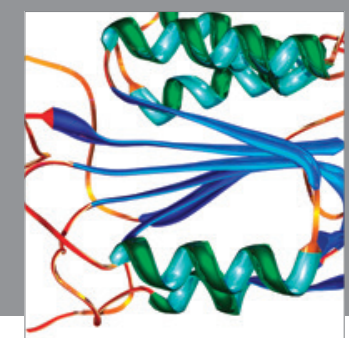

Disease Markers
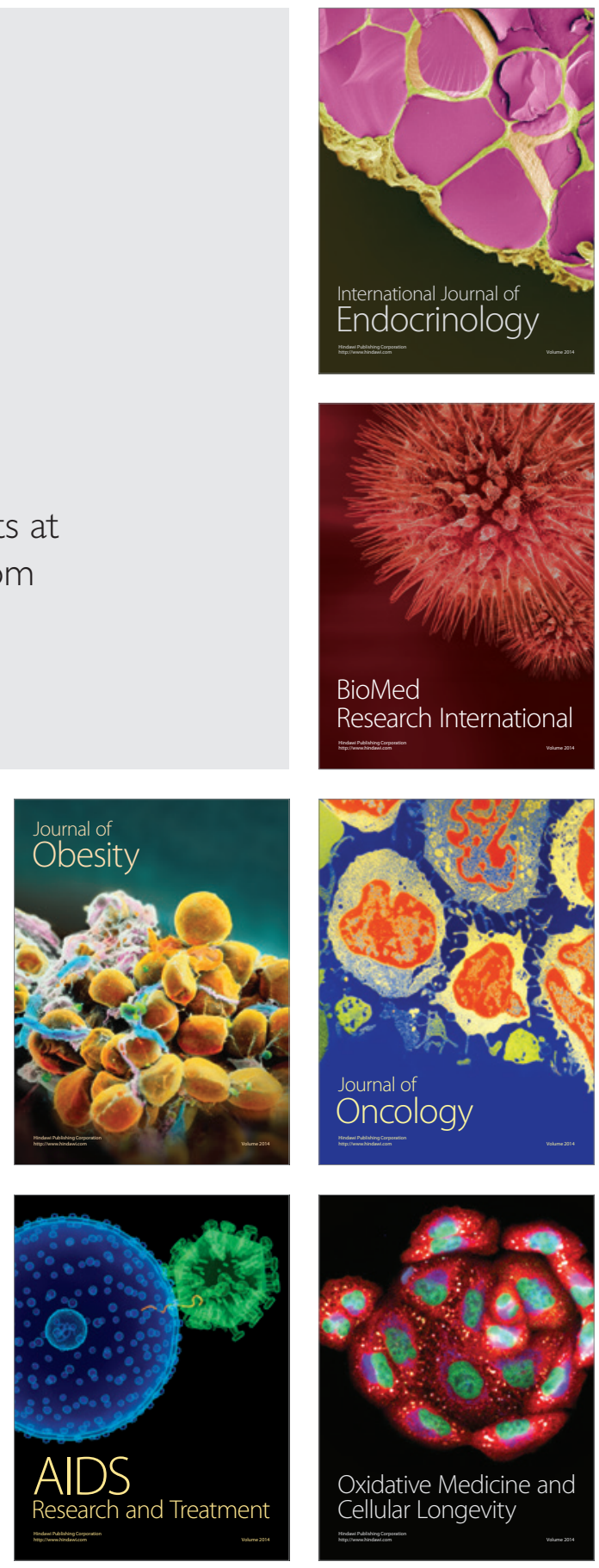